\title{
SOME REMARKS ON THE DECOMPOSITION OF KERNELS
}

\author{
ARNOLD JANSSEN
}

\begin{abstract}
In a recent paper $\mathrm{K}$. Lange has proved that the decomposition of a stochastic kernel into a continuous and discontinuous part yields kernels again. In the present paper, the author gives a short proof of this theorem and establishes a more general decomposition theorem. Finally, a counterexample shows that in general the Lebesgue decomposition of a kernel does not produce kernels.
\end{abstract}

K. Lange [4] applied the results of Dubins and Freedman [1] to the decomposition of kernels. Here is a short proof of Theorem 8 [4]. A counterexample shows, that in general the mentioned decompositions of kernels are no longer valid. Moreover, if the $\sigma$-field is not countably generated, Lemma 1 yields a decomposition of a kernel into a kernel which is concentrated on the atoms of a given countably generated sub- $\sigma$-field and a second kernel equal to zero on the atoms of the sub- $\sigma$-field.

Let $\Lambda$ be any $\sigma$-field over a set $X$ and $\Sigma$ a countably generated sub- $\sigma$-field of $\Lambda$. The spaces of finite (positive) measures $M(X, \Lambda)$ and $M(X, \Sigma)$ may carry the $\sigma$-fields $\Lambda^{*}$ and $\Sigma^{*}$, the coarsest $\sigma$-fields so that the functions $\mu \mapsto \mu(A)$ are measurable for all $A \in \Lambda$ and $\Sigma$ respectively (compare [1]). Every measure $\mu \in M(X, \Lambda)$ can uniquely be decomposed into a part $\mu_{1}$ which is concentrated on a countable number of atoms of the sub- $\sigma$-field $\Sigma$, and a part $\mu_{2}$, which is singular with respect to all measures with this property. For $A \in \Lambda$ let $\mu_{\mid A}$ denote the restriction of $\mu$ on $A$ and $\mu_{\mid \Sigma}$ the restriction of $\mu$ on the sub- $\sigma$-field. We can describe the value of the first part as $\mu_{1}(X)=\left(\mu_{\mid \Sigma}\right)_{1}(X)$ where on the right-hand side the index 1 denotes the atomic part of the restricted measure. Hence

$$
\mu_{1}(A)=\left(\mu_{\mid A}\right)_{1}(X)=\left(\left(\mu_{\mid A}\right)_{\mid \Sigma}\right)_{1}(X) \text { for } A \in \Lambda .
$$

LEMMA 1. The functions $\mu \mapsto \mu_{i}, i=1,2$, from $M(X, \Lambda)$ into $M(X, \Lambda)$ are $\left(\Lambda^{*}, \Lambda^{*}\right)$-measurable.

Proof. It suffices to show that $\mu \mapsto \mu_{1}(A)$ is $\Lambda^{*}$-measurable for all $A \in \Lambda$. The restrictions

$$
\begin{aligned}
& M(X, \Lambda) \rightarrow M(X, \Lambda) \quad \text { and } \quad M(X, \Lambda) \rightarrow M(X, \Sigma) \\
& \mu \mapsto \mu_{\mid A} \quad \mu \mapsto \mu_{\mid \Sigma}
\end{aligned}
$$

Received by the editors March 27, 1978 and, in revised form, May 8, 1978.

AMS (MOS) subject classifications (1970). Primary 28A20, 28A32; Secondary 60G05.

Key words and phrases. Stochastic kernels, decomposition theorem, distribution function. 
are $\left(\Lambda^{*}, \Lambda^{*}\right)$-measurable and $\left(\Lambda^{*}, \Sigma^{*}\right)$-measurable respectively. The function

$$
\begin{aligned}
M(X, \Sigma) & \rightarrow M(X, \Sigma) \\
\nu & \mapsto \nu_{1}
\end{aligned}
$$

is $\left(\Sigma^{*}, \Sigma^{*}\right)$-measurable [1, Theorem 2.12] $\left(\nu_{1}\right.$ is the atomic part of $\left.\nu\right)$. Hence $\mu \mapsto \mu_{\mid A} \rightarrow\left(\mu_{\mid A}\right)_{\Sigma} \mapsto\left(\left(\mu_{\mid A}\right)_{\Sigma}\right)_{1} \mapsto\left(\left(\mu_{\mid A}\right)_{\Sigma}\right)_{\mid 1}(X)=\mu_{1}(A)$ is measurable.

Following the arguments of [4], Lemma 1 can be applied to the decomposition of kernels. Let $(Y, \Gamma)$ be any measurable space and $y \mapsto \mu(y, \cdot)$ a kernel, i.e. a $\left(\Gamma, \Lambda^{*}\right)$-measurable function from $Y$ into $M(X, \Lambda)$. For fixed $y \in Y$ let $\mu(y, \cdot)=\mu_{1}(y, \cdot)+\mu_{2}(y, \cdot)$ be the decomposition considered in Lemma 1 . Plainly, $y \mapsto \mu_{i}(y, \cdot), i=1,2$, are kernels. We now give a short proof of a decomposition theorem of K. Lange [4, Theorem 8], without using the Fell topology. For a finite measure $\mu$ over $\left(\mathbf{R}^{n}, \mathfrak{B}^{n}\right)$ let $\mu_{1}$ be the part with discontinuous distribution (or $\mu_{1}=0$ ) and $\mu_{2}$ the part with continuous distribution function so that $\mu_{1}$ is singular with respect to all measures with continuous distribution. See [4].

ThEOREM 2 ([4, ThEOREM 8]). Let

$$
\begin{aligned}
Y & \rightarrow M\left(\mathbf{R}^{n}, \mathfrak{B}^{n}\right) \\
y & \mapsto \mu(y, \cdot)
\end{aligned}
$$

be a kernel and $\mu(y, \cdot)=\mu_{1}(y, \cdot)+\mu_{2}(y, \cdot)$ be the decomposition described above. Then $y \mapsto \mu_{j}(y, \cdot)(j=1,2)$ are kernels.

Proof. We show: $\mu \mapsto \mu_{2}$ is $\left(\mathfrak{B}^{n *}, \mathfrak{B}^{n *}\right)$ measurable. Choose $\Lambda=\mathfrak{B}^{n}, \Sigma_{i}=$ $\pi_{i}^{-1}(\mathscr{B})$, with $\pi_{i}$ the $i$ th projection. Let $\mu_{2}^{i}$ be the second part of the decomposition of Lemma 1 with $\mu_{2}^{i} \perp \nu$ for all $\nu$ which are concentrated on the hyperplanes given by $\pi_{i}^{-1}(\{c\})$ for $c \in \mathbf{R}$. The function $\varphi_{i}: \mu \rightarrow \mu_{2}^{i}$ is measurable. Hence $\varphi:=\varphi_{1} \circ \varphi_{2} \circ \cdots \circ \varphi_{n}$ is measurable and $\varphi(\mu)=\mu_{2}$. See [4, Theorem 3].

REMARK. The proof of Theorem 2 holds in a more general case. Let $\Sigma$ be countably generated and for an integer $n$ let $\bigotimes_{i=1}^{n} \Sigma$ denote the product- $\sigma$-field on the product space $\times_{i=1}^{n} X$. It may be assumed that the atoms of $\Sigma$ consist only of single points $x \in X$. Then the decomposition of a kernel into a part which lies on the sets $\pi_{i}^{-1}(\{x\}), i=1, \ldots, n, x \in X$, and a second part equal to zero on those sets yields kernels.

EXAMPLE. Let $f$ be a measurable function from an arbitrary measurable space $(X, \Lambda)$ into a countably generated space $\left(Z, \Sigma^{\prime}\right)$. In the situation of Lemma $1 \Sigma$ can be chosen as $f^{-1}\left(\Sigma^{\prime}\right)$.

For example let $f$ from $\mathbf{R}^{n}$ to $R$ be the euclidean norm. Then for every kernel it is possible to split off that part of the kernel which gives positive mass to spheres centered at that origin.

COUNTEREXAMPLES. For countably generated measurable spaces K. Lange [4] has proved a Lebesgue decomposition theorem for kernels. Here is an example which shows that this theorem is not true in general. 
Choose $\mathbf{R}$ and the $\sigma$-field $\Lambda=\left\{A \subset \mathbf{R}: A\right.$ or $A^{c}$ countable $\}$. By definition, let $\nu$ be the measure over $(\mathbf{R}, \Lambda)$ with the property that $\nu(A)=1$ if $A^{c}$ is countable and $\nu(A)=0$ otherwise. Choose a set $E \subset \mathbf{R}$ with $E \notin \Lambda$. If we denote by $\delta_{y}$ the Dirac measure in the point $y$ and by $I_{E}$ the indicator of $E$, the function $y \mapsto \mu(y, \cdot)=I_{E}(y) \delta_{y}+I_{E} c(y) \nu$ from $(\mathbf{R}, \Lambda)$ into $\left(M(\mathbf{R}, \Lambda), \Lambda^{*}\right)$ is a kernel, since $y \mapsto \mu(y, A)$ is $\Lambda$-measurable for all $A \in \Lambda$.

The absolutely continuous part of the Lebesgue decomposition of $\mu(y, \cdot)$ with respect to $\nu$ is $\mu_{1}(y, \cdot)=I_{E} c(y) \nu(\cdot)$. The function $y \mapsto \mu_{1}(y, \cdot)$ is not a kernel, as $\mu_{1}(y, \mathbf{R})=I_{E} c(y)$.

The decomposition of a kernel into a discrete part and another part, whose measures are singular with respect to all measures with this property does not in general yield two kernels. Choose the kernel $\mu(y, \cdot)$ as above. Then the discrete part $I_{E}(y) \delta_{y}$ is not a kernel. In [3] $\mathrm{K}$. Lange deals with $\Lambda^{*}$ measurable sets of measures. Let $L=\left\{\delta_{y}: y \in \mathbf{R}\right\} \cup\{\nu\}$ carry the restricted $\sigma$-field $\Lambda_{\mid L}^{*}$.

The sets $\left\{\delta_{y}: y \in \mathbf{R}\right\}$ and $\{\nu\}$ are not $\Lambda_{\mid L}^{*}$-measurable subsets of $L$. If for example $I_{\{v\}}: L \rightarrow \mathbf{R}$ were $\Lambda_{\{L}^{*}$-measurable, so would $y \rightarrow I_{\{v\}}(\mu(y, \cdot))=$ $I_{E} c(y)$ be measurable-a contradiction. The sets of measures $\{\mu \in M(\mathbf{R}, \Lambda)$ : $\mu \ll \nu\}$ and $\{\mu \in M(\mathbf{R}, \Lambda): \mu \perp \nu\}$ are not $\Lambda^{*}$-measurable, since their intersection with $L$ is not measurable with respect to the restricted $\sigma$-field. The second set is the set of discrete (finite) measures.

Other results on the decomposition of kernels will appear in [2].

I am grateful to Professor Dr. Plachky for several helpful discussions.

\section{REFERENCES}

1. L. Dubins and D. Freedman, Measurable sets of measures, Pacific J. Math. 14 (1964), 1211-1222. MR 30 \# 4887.

2. A. Janssen, Uber die Messbarkeit der Mengen der zulässigen und singulären Translationen von Massen; der Lebesguesche Zerlegungssatz für Kerne, Lecture Notes, Oberwolfach 1978, Wahrscheinlichkeitsmasse auf Gruppen (to appear).

3. K. Lange, Borel sets of probability measures, Pacific J. Math. 48 (1973), 141-161. MR 50 \# 10191.

4. K. Lange, Decomposition of substochastic transition functions, Proc. Amer. Math. Soc. 37 (1973), 575-580.

abteilung Mathematix, Universitït Dortmund, Postfach 5005 00, D-4600 Dortmund 50, Federal Republic OF Germany 